\title{
Toy models for the falling chimney
}

\author{
Gabriele Variesch:* and Kaoru Kamiyat \\ Department of Physics, Loyola Marymount University, \\ One LMU Drive, Los Angeles, CA 90045
}

(Dated: February 2, 2008)

\begin{abstract}
In this paper we review the theory of the "falling chimney", which deals with the breaking in mid-air of tall structures, when they fall to the ground. We show that these ruptures can be caused by either shear forces, typically developing near the base, or by the bending of the structure, which is caused primarily by the internal bending moment.

In the latter case the breaking is more likely to occur between one third and one half of the height of the chimney. Small scale toy models are used to reproduce the dynamics of the falling chimney. By examining photos taken during the fall of these models we test the adequacy of the outlined theory. This type of experiment, easy to perform and conceptually challenging, can become part of a rotational mechanics lab for undergraduate students.
\end{abstract}




\section{INTRODUCTION}

One of the most interesting demonstrations for an introductory mechanics course is the "Falling Chimney - Free Fall Paradox," as it was named by Sutton in his classical book Demonstration Experiments in Physics. $\stackrel{1}{=}$ In the original version of this demonstration a ball is placed at one end of a uniform stick, which is pivoted at the other end and makes initially an angle of about $30^{\circ}$ with the horizontal. The elevated end of the stick is suddenly dropped, together with the ball, thus showing a very counter-intuitive behavior. The falling end of the stick accelerates at a greater rate than the free-falling ball, proving that its acceleration is greater than $g$, the acceleration of gravity.

A simplified version of the experiment can be performed just with a meter stick and a coin. The stick is supported in the horizontal position by two fingers, placed near the two ends. A coin is set on the stick near one end, which is suddenly released. The effect is similar to the previous demonstration: the falling end of the rotating stick eventually acquires an acceleration greater than that of the freely falling coin, which loses contact with the stick surface and lags behind the falling stick.

A description of the first version of the experiment can be found in almost every book of physics demonstrations, ${ }^{2,3.4}$ sometimes with different names ("Falling Stick", "Hinged Stick and Falling Ball", and others). Photographic descriptions or even video-clips of this demo can be found on-line in several web-pages (see our web-page, $\frac{5}{5}$ for a collection of related links).

In addition, countless papers exist in the literature; we have traced several of these, from the 1930 's to the present. Some of the earliest discussions can be found in Constantinides 6 and Ludeke ${ }^{7}$ (as well in the book by Sutton ${ }^{1}$ ), followed by many others. ${ }^{8.9 .10 .11 .12}$ These concentrate mostly on the simple explanation of the effect, which relies on the concept of "center of percussion" of the rotating stick (a simple introduction to this concept can be found in Bloomfield ${ }^{13}$ ). This particular point of the stick (located at a distance from the hinged end equal to two thirds of the length, for a uniform stick) is moving with the same acceleration as a particle under gravity, constrained to move along the same circular path. Points on the stick beyond the center of percussion descend with accelerations greater than that of particles freely moving under gravity, on their respective circular paths. As a consequence of this, if the initial angle formed by the stick with the horizontal is less than about $35^{\circ}$, the end point will possess at all times a vertical component of the acceleration greater than $g$, producing the effect described above.

Several variations of the basic demonstration also exist, $\stackrel{14.15 .16 .17 .18 .19}{1}$ the majority of which suggest attaching an additional mass to the rotating stick at different positions. The effect for the student or the viewer is even less intuitive than the original version: an additional mass placed near the end of the stick actually reduces the acceleration of the end point, affecting substantially the outcome of the experiment. In general the addition of a mass at any point on the stick will increase both the total torque on the system (thus increasing the rotational acceleration) and the moment of inertia of the system around the axis of rotation (resulting in a decreased rotational acceleration). The center of percussion of the stick still plays a key role: if the additional mass is placed beyond it, the effect of the increased moment of inertia dominates and the acceleration of the rotational motion will be reduced. If the mass is placed before the center of percussion, the increase in the torque will dominate and the rotational motion will be enhanced. The effect is null if the mass is placed exactly at

the center of percussion (a complete discussion of this effect can be found in Bartlett ${ }^{17}$ and 
Haber-Schaim 19 ).

The next logical step is to analyze the behavior of a real falling chimney. Almost invariably a tall chimney, falling to the ground like the stick in the previous discussion, will break in mid-air at some characteristic height. This is well documented in several photos reproduced in the literature, such as the one which appeared on the cover of the September 1976 issue of The Physics Teacher (other photos can be found in Bundy ${ }^{20}$ and Bartlett, ${ }^{21}$ or also on our web-page ${ }^{5}$ ). The causes of such breaking, the height of the rupture point and the angle at which the breaking is most likely to occur, are the most natural questions which arise.

The first analysis ${ }^{22}$ of this problem compared the fall of the real chimney to the fall of the hinged stick, but wrongly identified the center of percussion (at about two thirds of the height) as the probable point of rupture. Reynolds ${ }^{23}$ first identified the possible causes of the breaking with the shear forces and the bending moment originating within the structure of the toppling chimney. More detailed analyses were given by Bundy ${ }^{20}$ and $\operatorname{Madsen}^{24}$ (the most complete papers we found on the subject) while simplified explanations are also reported. ${ }^{21,25,26}$ It even appears in graduate student study guides,${ }^{27.28}$ although the chimney is shown bending the wrong way in one of these books.

In this paper we review the theory of the real falling chimney, outlined by Madsen, ${ }^{24}$ aiming for a complete and clear explanation of this phenomenon in Sects. II and III. Then, in Sect. IV] we propose simple ways of using small scale models (literally toy models - made with toy blocks and bricks) to test effectively the outlined theory. More information on these toy models can also be found on our web-site, $\frac{5}{2}$ together with photos and movie clips of the experiments we have performed.

\section{ROTATIONAL MOTION OF THE FALLING CHIMNEY}

The rotational motion of a falling chimney under gravity is equivalent to that of the falling hinged stick of Sect. I. We can describe it as in Fig. 11 where we use polar coordinates $r$ and $\theta$ (with $\widehat{e}_{r}$ and $\widehat{e}_{\theta}$ as unit vectors) for the position of an arbitrary point $A$ on the longitudinal axis of the chimney, measuring the angle $\theta$ from the vertical direction. We treat the chimney as a uniform rigid body of mass $m$ and height $H$, under the action of its weight $\mathbf{W}=m \mathbf{g}$, applied to the center of gravity (basically the center of mass-CM- of the body), and a force $\mathbf{F}$ exerted by the ground on the base of the chimney, assumed to act at a single point (we neglect air resistance, or any other applied force). In plane polar coordinates:

$$
\begin{aligned}
\mathbf{W} & =W_{r} \widehat{e}_{r}+W_{\theta} \widehat{e}_{\theta}=-m g \cos \theta \widehat{e}_{r}+m g \sin \theta \widehat{e}_{\theta} \\
\mathbf{F} & =F_{r} \widehat{e}_{r}+F_{\theta} \widehat{e}_{\theta} .
\end{aligned}
$$

The moment of inertia of the chimney can be approximated with the one for a uniform thin rod, with rotation axis perpendicular to the length and passing through one end: ${ }^{35}$

$$
I=\frac{1}{3} m H^{2} \text {. }
$$

Applying the torque equation $I \ddot{\theta}=\tau_{z}$, for a rotation around the origin, with an external torque given by $\tau_{z}=m g \frac{H}{2} \sin \theta$, we find the angular acceleration

$$
\ddot{\theta}=\frac{\tau_{z}}{I}=\frac{3}{2} \frac{g}{H} \sin \theta .
$$




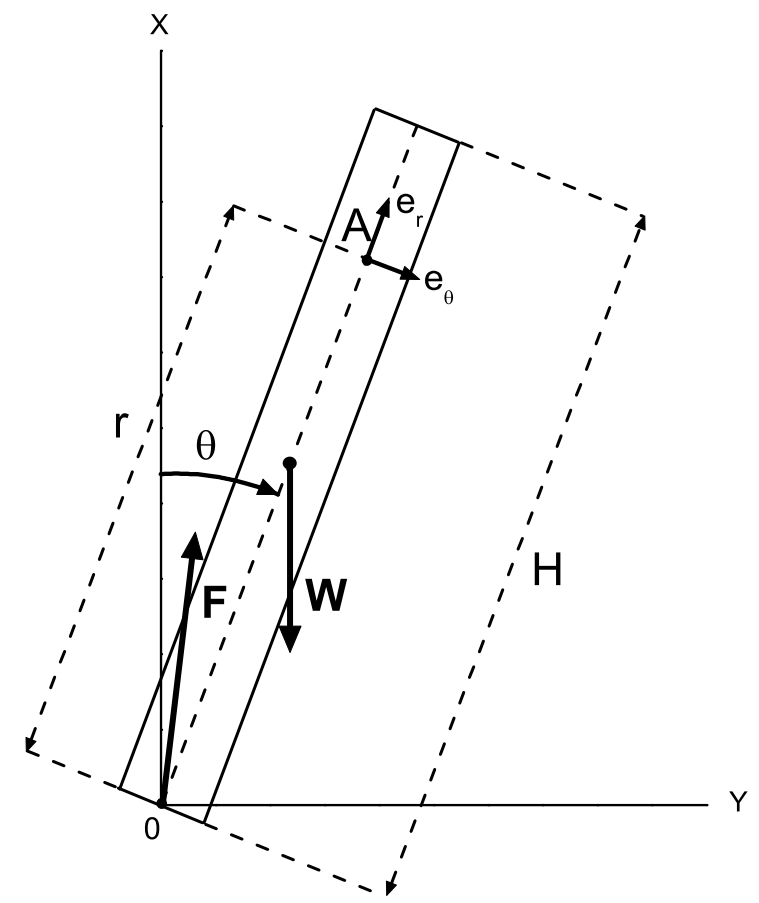

FIG. 1: The falling chimney described as a rotating uniform stick. The external forces are the weight of the body applied to the center of gravity, and the constraint force at the base.

A simple integration, using $\ddot{\theta}=\frac{d \dot{\theta}}{d t}=\frac{d \dot{\theta}}{d \theta} \dot{\theta}=\frac{3}{2} \frac{g}{H} \sin \theta$, gives the angular velocity

$$
\dot{\theta}^{2}=3 \frac{g}{H}(1-\cos \theta),
$$

assuming that the chimney starts moving from rest and is initially in the vertical direction. A further integration of Eq. 4, can lead to $\theta(t)$ in terms of elliptic integrals.

We recall that the acceleration in polar coordinates can be written as $\mathbf{a}=\ddot{\mathbf{r}}=\left(\ddot{r}-r \dot{\theta}^{2}\right) \widehat{e}_{r}+$ $(r \ddot{\theta}+2 \dot{r} \dot{\theta}) \widehat{e}_{\theta}$, so that, for a point $A$ at a fixed distance $r$ from the origin, it becomes

$$
\mathbf{a}=a_{r} \widehat{e}_{r}+a_{\theta} \widehat{e}_{\theta}=-r \dot{\theta} \widehat{e}_{r}+r \ddot{\theta} \widehat{e}_{\theta} .
$$

For a point at two thirds of the height, $r=\frac{2}{3} H$, combining Eqs. 3 and 5 we get $a_{\theta}(r=$ $\left.\frac{2}{3} H\right)=\frac{2}{3} H \ddot{\theta}=g \sin \theta$, proving that this particular point is the center of percussion of the body, as already mentioned in Sect. I.

The torque equation allowed us to determine the angular acceleration of the motion in Eq. 3. We can use this result and Newton's second law for the motion of the center of mass $(\mathrm{CM})$ of the whole chimney to determine the unknown force $\mathbf{F}$ at the base. The vectorial equation is

$$
m \ddot{\mathbf{r}}_{C M}=\mathbf{W}+\mathbf{F}
$$




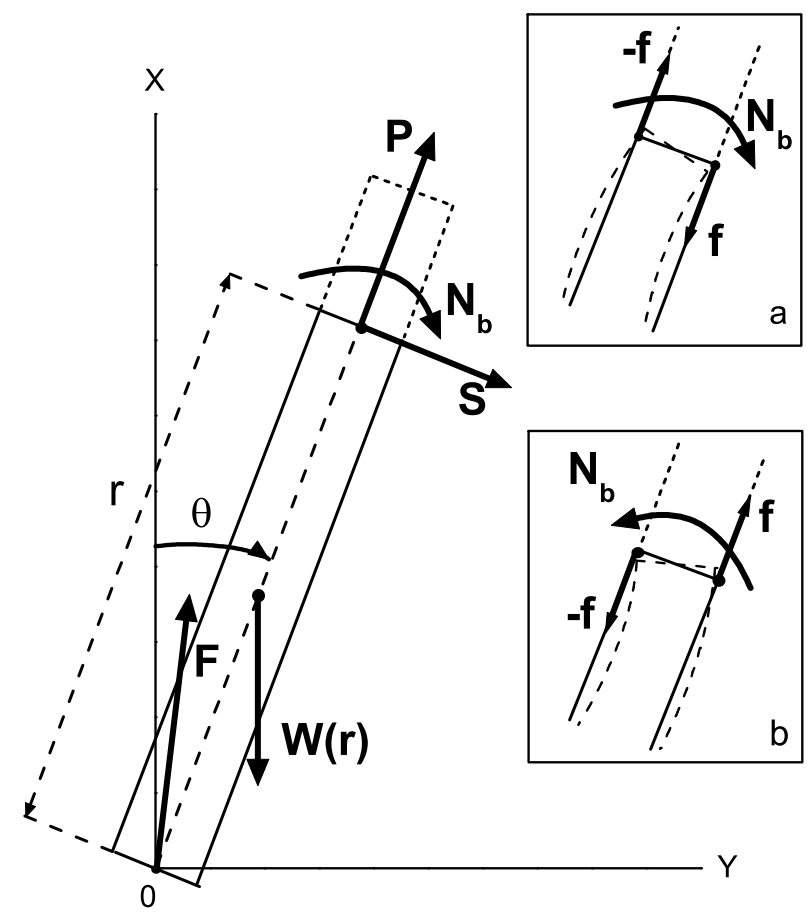

FIG. 2: The forces acting on the lower portion of the chimney, due to the upper part and the action of the constraint at the base, are shown here. The two insets explain the definition of the bending moment in terms of a couple of forces. The resulting deformation of the structure is also shown for the two possible cases.

which, for $r=\frac{H}{2}$, splits into radial and angular equations,

$$
\begin{aligned}
-m \frac{H}{2} \dot{\theta}^{2} & =F_{r}-m g \cos \theta \\
m \frac{H}{2} \ddot{\theta} & =F_{\theta}+m g \sin \theta .
\end{aligned}
$$

Using Eqs. 3] and 4, the two components of the force $\mathbf{F}$ are easily determined:

$$
\begin{aligned}
& F_{r}=\frac{5}{2} m g\left(\cos \theta-\frac{3}{5}\right) \\
& F_{\theta}=-\frac{1}{4} m g \sin \theta .
\end{aligned}
$$

\section{INTERNAL FORCES AND BENDING MOMENT}

We now move to the analysis of the internal forces which develop inside the structure of the falling chimney. The resulting stresses and bending moment are the causes of the rupture of the toppling chimney. Consider, as in Fig. 2, an arbitrary lower portion of the chimney of height $r$ (as opposed to the total height $H$ ) and the forces acting on this part of the structure due to the upper portion and the base. The weight of the lower portion is now 
$W(r)=m g \frac{r}{H}$ (assuming again a uniform structure, so that the weight is proportional to the height of the considered portion) and it is applied to the center of gravity of this lower portion (at a distance $\frac{r}{2}$ from the origin). In polar coordinates:

$$
\mathbf{W}(r)=W_{r}(r) \widehat{e}_{r}+W_{\theta}(r) \widehat{e}_{\theta}=-m g \frac{r}{H} \cos \theta \widehat{e}_{r}+m g \frac{r}{H} \sin \theta \widehat{e}_{\theta} .
$$

The force $\mathbf{F}$, applied at the base, is still the same as in Eqs. 8a, 8b, but we have to add the action of the upper part on the lower portion. We follow here the general analysis of the internal forces and moments which can be found in every textbook on Statics (see for example ${ }^{29.30}$ ) and which can be easily adapted to our case.

The distribution of all the internal forces, at the cross section being considered, can be equivalently described by a resultant force and a resultant moment acting at a specific point of the cross section (typically the "centroid" of the sectioned area, in our case simply the central point of the section, on the longitudinal axis). In particular, the resultant force can be decomposed into a transverse shearing force $\mathbf{S}=S_{\theta} \widehat{e}_{\theta}$, and a longitudinal stress force (tension or compression) $\mathbf{P}=P_{r} \widehat{e}_{r}$, applied as in Fig. 2, at the cross section between the upper and lower portions, and assumed positive in the $\widehat{e}_{\theta}, \widehat{e}_{r}$ direction respectively.

In addition, we have to consider the resultant moment of the forces at the cross section, which is usually called the "bending moment" $\mathbf{N}_{b}$, because its effect will ultimately result in bending the structure. It is represented in the picture by the curved arrow. Since we treat this as a plane problem, the bending moment can only have a component perpendicular to the plane of the figure, i.e., in the $z$ direction. No other components are considered here, in particular we assume that no torsional moment exist in the structure, which would tend to twist the chimney around its longitudinal axis.

The bending moment $\mathbf{N}_{b}=N_{b} \widehat{e}_{z}$ can be thought as originating from a couple of forces, $\mathbf{f}$ and $-\mathbf{f}$, that can be regarded as applied to the leading and trailing edge of the structure, at the cross section considered. This couple of forces is shown explicitly in the papers by Bundy ${ }^{20}$ and Madsen, ${ }^{24}$ but we prefer to use directly $\mathbf{N}_{b}$ in our treatment, because the bending moment is the result of the whole distribution of forces at the cross section considered. The two small insets inside Fig. 2] explain the definition of the bending moment in terms of the couple of forces $\mathbf{f}$ and $\mathbf{- f}$. We also show the resulting deformation of the structure due to a "clockwise" (diagram a), or a "counter-clockwise" bending moment (diagram b). The latter case will be the actual deformation of the falling chimney. $N_{b}$ will be assumed to be positive if it acts as in the figure, i.e., a positive component of the torque in the $z$ direction (we assume here the use of a right-handed system of coordinate axis). In the following we will refer to $N_{b}$ as the bending moment, acting on the lower portion of the chimney. ${ }^{36}$

Again, we will consider the torque equation and the second law for the motion of the center of mass (located at $\frac{r}{2}$ ) for just the lower portion of the chimney (of mass $\frac{r}{H} m$ ). It is better to analyze the CM motion first. The vector equation

$$
m \frac{r}{H} \ddot{\mathbf{r}}_{C M}=\mathbf{W}(r)+\mathbf{F}+\mathbf{P}+\mathbf{S}
$$

will split into the radial and angular directions,

$$
\begin{aligned}
-m \frac{r^{2}}{2 H} \dot{\theta}^{2} & =-\frac{3}{2} m g(1-\cos \theta) \frac{r^{2}}{H^{2}}=-m g \frac{r}{H} \cos \theta+\frac{5}{2} m g\left(\cos \theta-\frac{3}{5}\right)+P_{r} \\
m \frac{r^{2}}{2 H} \ddot{\theta} & =\frac{3}{4} m g \sin \theta \frac{r^{2}}{H^{2}}=m g \frac{r}{H} \sin \theta-\frac{1}{4} m g \sin \theta+S_{\theta},
\end{aligned}
$$


having used Eqs. 3 4, 8a, 8b, and 9, We can solve for the longitudinal and transverse forces

$$
\begin{aligned}
& P_{r}=-\frac{1}{2} m g\left(1-\frac{r}{H}\right)\left[\left(5+3 \frac{r}{H}\right) \cos \theta-3\left(1+\frac{r}{H}\right)\right] \\
& S_{\theta}=\frac{3}{4} m g \sin \theta\left(\frac{r^{2}}{H^{2}}-\frac{4}{3} \frac{r}{H}+\frac{1}{3}\right),
\end{aligned}
$$

which depend on the fraction of height $\frac{r}{H}$, the angle of rotation $\theta$, and also the total weight $m g$. Following the analysis by Bundy, $\underset{20}{2}$ we plot these two forces in Figs. 3 and 4 respectively, normalized to the total weight $m g$, as a function of the height fraction, for several angles.

From Fig. [3 we see that $P_{r}$ is negative (a compression) for smaller angles, but eventually becomes positive (a tension) for angles greater than about $45^{\circ} . P_{r}$ also depends critically on $\frac{r}{H}$ (for $\theta=0^{\circ}, P_{r}$ represents simply the compression due to the weight of the upper part acting on the lower part). This longitudinal force will be combined later with the bending moment to determine the total stress at the leading and trailing edges, which is the most typical cause of the rupture.

In Fig. [ we plot the (transverse) shear force $S_{\theta}$, which can be the other leading cause of rupture. It is easily seen that, for any considered angle, the magnitude of the shear force $\left|S_{\theta}\right|$ has an absolute maximum at $\frac{r}{H}=0$ (and a positive value), meaning that large shear forces, in the $\widehat{e}_{\theta}$ direction, usually originate near the base. The shear force is always zero at one third of the height, and $\left|S_{\theta}\right|$ also has a (relative) maximum at $\frac{2}{3} H$ (with a negative value, therefore $S_{\theta}$ is in the $-\widehat{e}_{\theta}$ direction), but this value is smaller than the one near the base.

From this analysis, it is typically concluded that if the structure breaks from shear stress alone, this is usually more likely to happen near the base. This can be seen for example in the already mentioned cover photo of the September 1976 issue of The Physics Teacher,,$\frac{5,21}{2}$ showing the fall of a chimney in Detroit. The two ruptures at the bottom are likely due to shear forces, while a third rupture can be seen at about $r=0.47 \mathrm{H}$, and this is due to the combination of bending moment and longitudinal force $P_{r}$, as we will explain in the following. More photos and detailed pictorial descriptions of chimney ruptures can be found in the paper by Bundy 5.20

The "bending moment" $N_{b}$ can be calculated from the torque equation $I(r) \ddot{\theta}=\tau_{z}$, where now $I(r)=\frac{1}{3} m \frac{r}{H} r^{2}$ is the moment of inertia of just the lower part. $\ddot{\theta}$ will come from Eq. 3 , and the total external torque is now $\tau_{z}=\frac{r}{2} W_{\theta}(r)+r S_{\theta}+N_{b}$. Using Eqs. 9 and 12b, we can solve the torque equation for the bending moment, obtaining

$$
N_{b}=-\frac{1}{4} m g \sin \theta r\left(1-\frac{r}{H}\right)^{2},
$$

or, in a non-dimensional form

$$
\frac{N_{b}}{m g H}=-\frac{1}{4} \sin \theta \frac{r}{H}\left(1-\frac{r}{H}\right)^{2},
$$

which is plotted in Fig. 5, as a function of the height fraction and for various angles.

$N_{b}$ is always negative, showing that it is actually directed in the opposite way of Fig. 2 (or as in diagram b of Fig. 22). This particular direction of the bending moment will induce a tension in the leading edge of the chimney and a compression in the trailing edge. The 


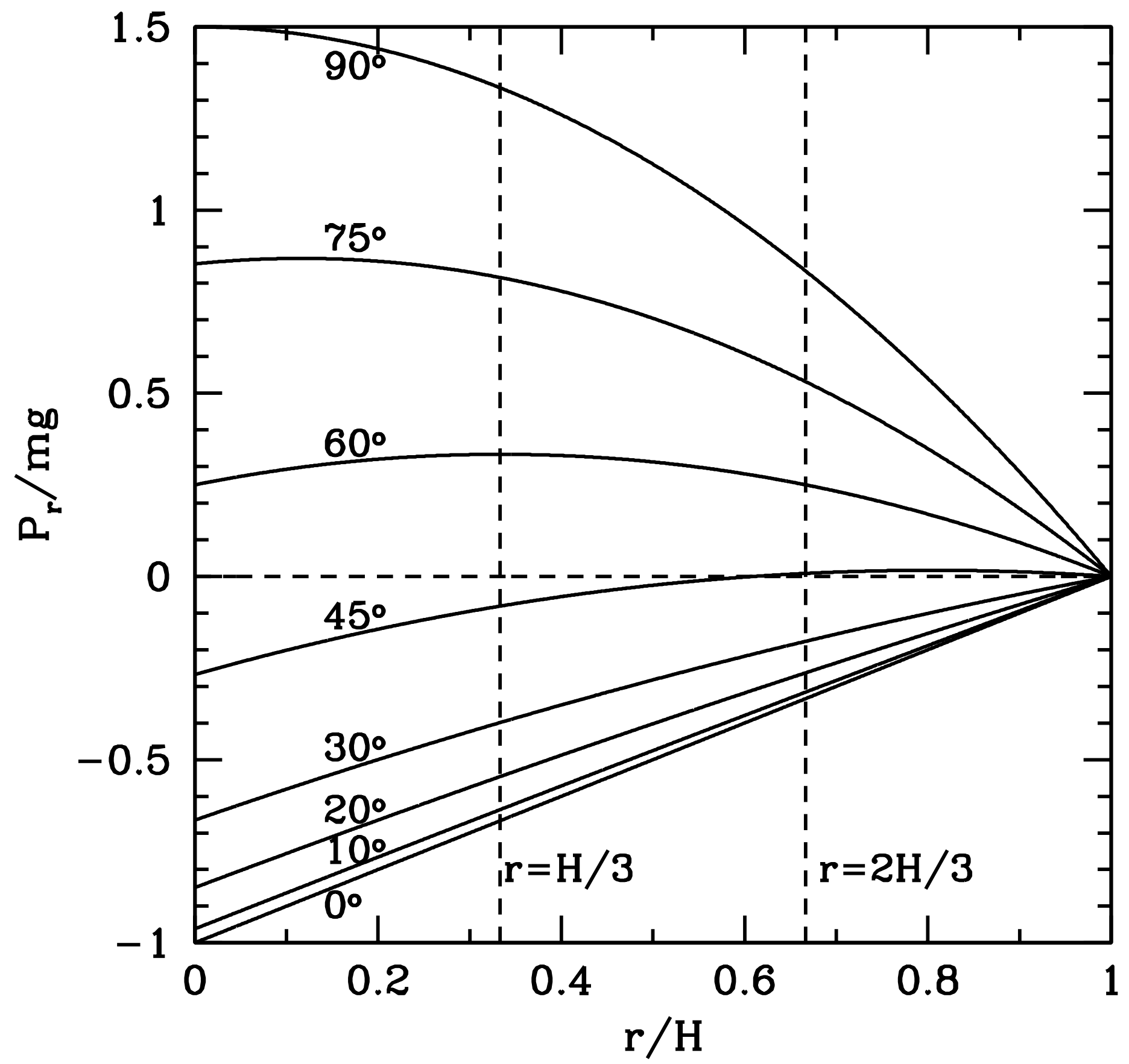

FIG. 3: The longitudinal stress force per unit weight of the chimney is shown as a function of the height fraction for several angles. Positive values indicate tensions, while negative values represent compressions. 


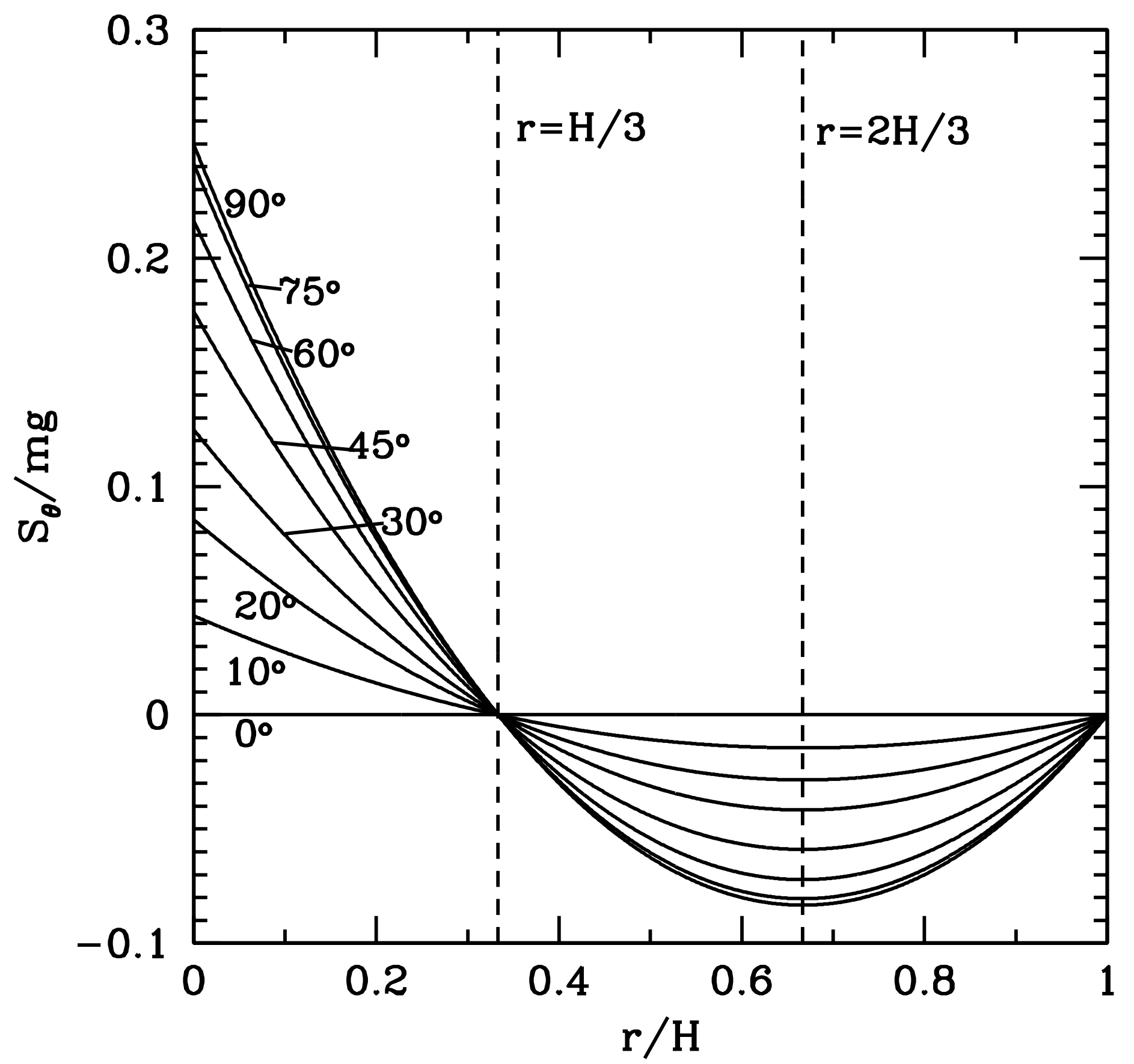

FIG. 4: The transverse shear force per unit weight of the chimney is shown as a function of the height fraction for several angles. Positive values are for forces in the $\widehat{e}_{\theta}$ direction. 


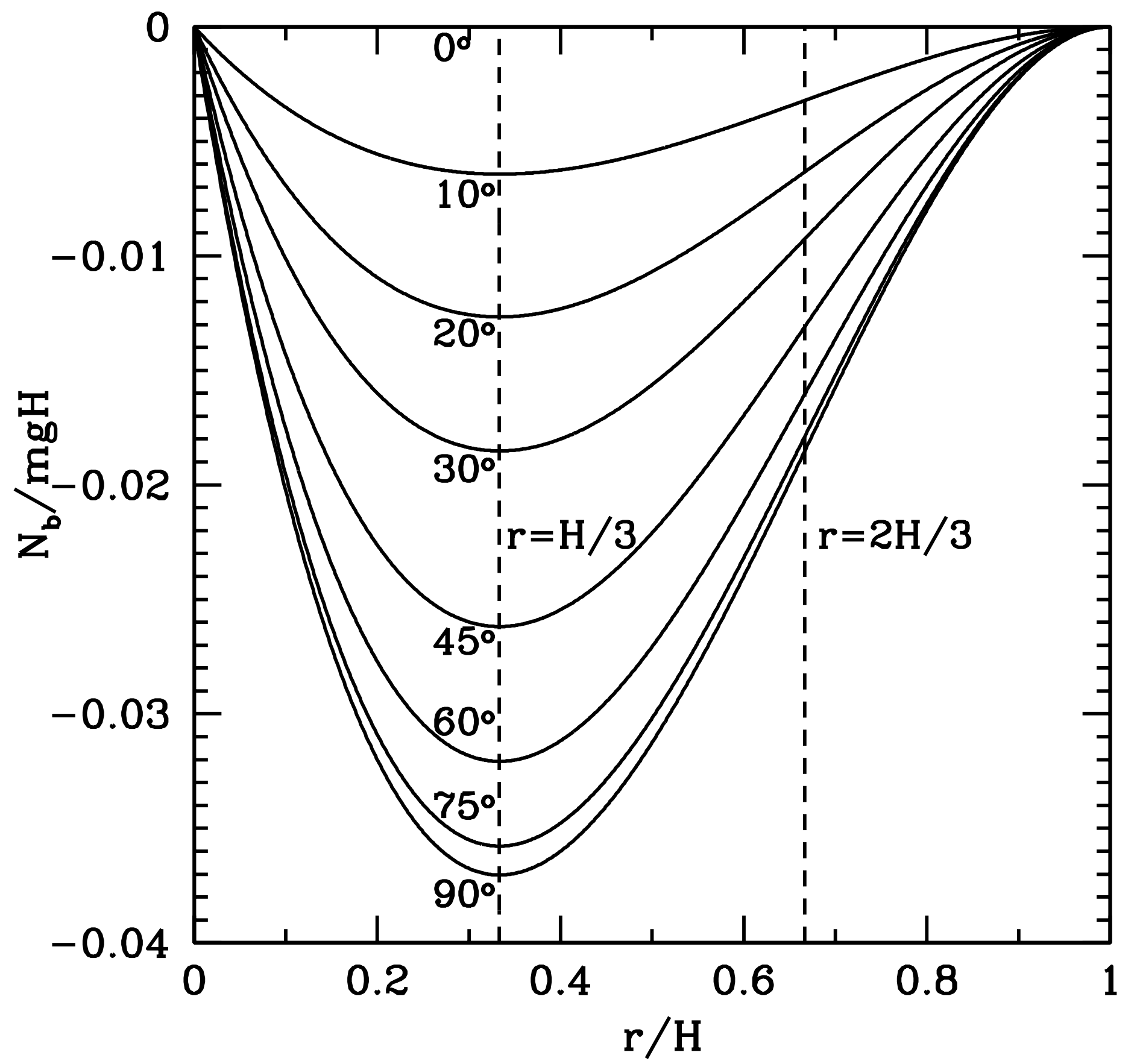

FIG. 5: The bending moment, divided by the weight and the height of the chimney, is shown as a function of the height fraction for several angles. 
structure will bend accordingly, with the concavity on the side of the trailing edge, and will eventually break in the way shown by the many existing photos. We can see that, for any angle, the bending moment is obviously zero at the bottom and at the top of the chimney, while it assumes its maximum absolute value at exactly one third of the height $H$. The bending moment alone would therefore induce a rupture at one third of the structure, but the total longitudinal stress at the leading edge is also due to the force $P_{r}$, as we will show next.

Another interesting relation between the bending moment $N_{b}$ and the shear force $S_{\theta}$ is that they are in general related by a simple derivative, i.e., $S_{\theta}=-\frac{\partial N_{b}}{\partial r}$, as it is easy to check from Eq. $12 \mathrm{~b}$ and Eq. [13 ${ }^{37}$ This is a well known relationship of the Statics of beams and other structural members (for a complete proof see for example Hibbeler ${ }^{30}$ ). It is a direct consequence of the equilibrium equations applied to an infinitesimal longitudinal portion of the beam: the change in bending moment along the beam is always equal to the shear force applied to that portion of the beam.

Finally, we can combine $N_{b}$ and $P_{r}$ to compute the total longitudinal stress on the cross sectional area between the lower and upper parts. We follow the theory of elasticity and deformations in beams, which can be found in classical treatises such as Sommerfeld ${ }^{31}$ and Landau-Lifshitz, 32 or again in Bundy's paper ${ }^{20}$ The longitudinal stress is maximum at the leading and trailing edges, located at the maximum distance from the longitudinal (centroidal) axis of the chimney which lies within the "neutral surface" of the structure, the surface which is neither stretched nor compressed.

For simplicity, we will only consider from here on, structures with uniform square cross section of side $a$, as this is the case of the toy models described in Sect. [V] In this case the stresses at the leading and trailing edge, $\sigma_{L}$ and $\sigma_{T}$ respectively, can be evaluated from

$$
\sigma_{L / T}=\frac{P_{r}}{A} \mp \frac{a N_{b}}{2 J}
$$

(the upper sign is for $\sigma_{L}$, the lower for $\sigma_{T}$ ) where $A=a^{2}$ is the area of the square cross section of side $a$, with the factor $\frac{a}{2}$ representing the distance between the longitudinal axis, considered as the "neutral axis," and the two edges. $J=\frac{a^{4}}{12}$ is the moment of inertia of the cross sectional area computed about the neutral axis (see Sommerfeld ${ }^{31}$ for details). Using also the expressions for $P_{r}$ and $N_{b}$, we obtain

$$
\frac{\sigma_{L / T} a^{2}}{m g}=-\frac{1}{2}\left(1-\frac{r}{H}\right)\left[\left(5+3 \frac{r}{H}\right) \cos \theta-3\left(1+\frac{r}{H}\right)\right] \pm \frac{3}{2} \frac{H}{a} \sin \theta \frac{r}{H}\left(1-\frac{r}{H}\right)^{2}
$$

where we normalized $\sigma_{L / T}$, dividing by $\frac{m g}{a^{2}}$, in order to obtain a dimensionless quantity which is plotted in Fig. 6, as a function of the height fraction and for several angles.

This quantity depends also on the ratio $\frac{H}{a}$, which for a real chimney is of the order $\frac{H}{a} \gtrsim 10$. For the toy models described in Sect. IV] the value of this ratio is even bigger: $\frac{H}{a} \simeq 24-61$, enhancing the contribution of the second term of Eq. 16, which comes from the bending moment $N_{b}$. In Fig. 6] we show the plot for $\frac{H}{a}=24$, but similar figures can be obtained for different values of the ratio.

In Eqs. 15, 16, and in Fig. 6 the total stresses are considered positive if they represent tensions, negative if they are compressions. It is easily seen from the figure that the stress at the leading edge $\sigma_{L}$, is initially a compression, but eventually becomes a tension, constantly increasing for larger angles; $\sigma_{T}$ on the contrary is usually a compression. It is therefore the 


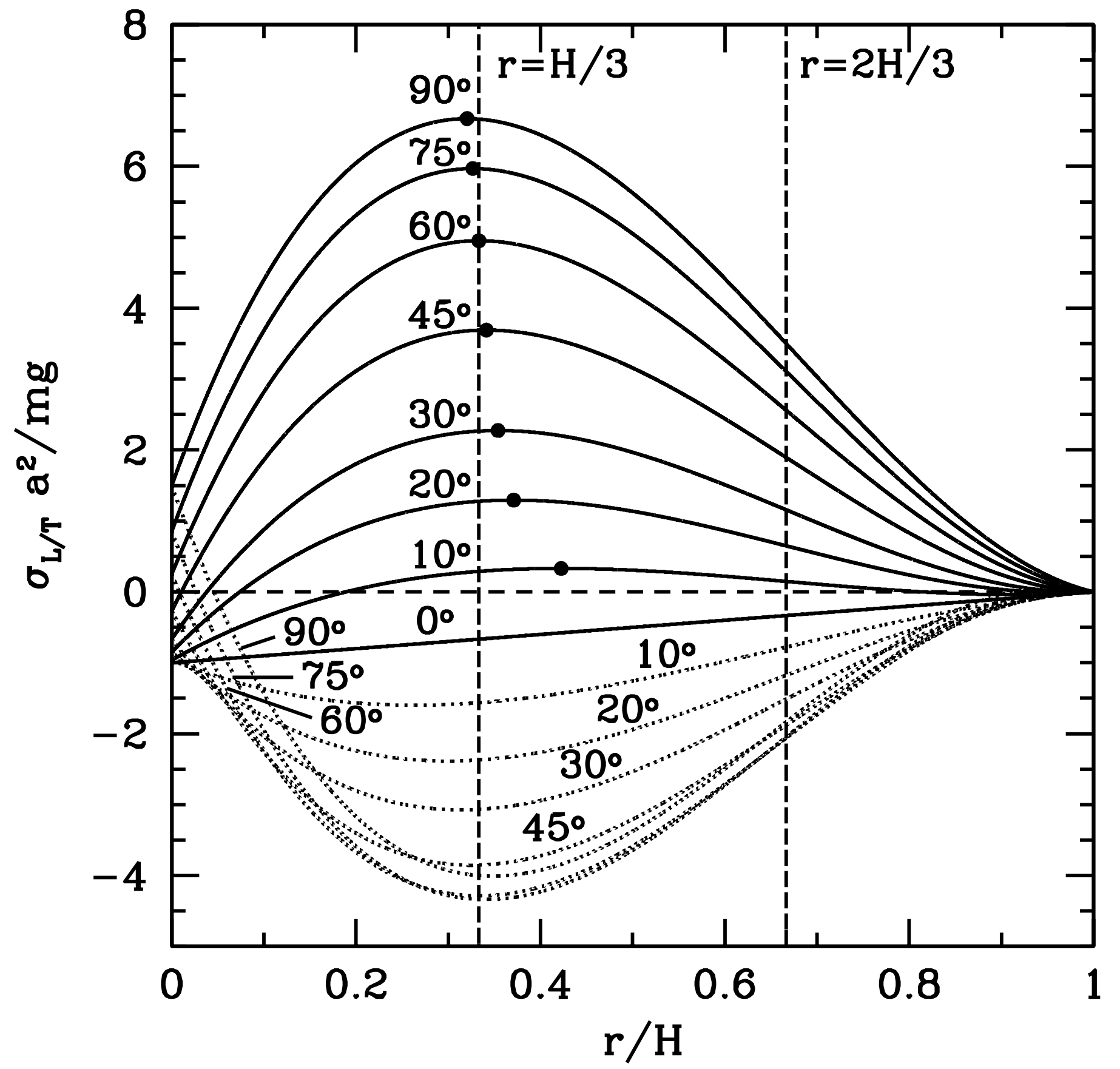

FIG. 6: The normalized longitudinal stress at the leading edge (solid) and at the trailing edge (dotted), are shown as a function of the height fraction and for several angles $(H / a=24)$. The maxima of the leading edge stress curves are marked by solid points. 
combination of these intense tensile stresses in the leading part of the chimney (and also compressions on the trailing side) that causes the rupture of the chimney.

This type of breaking is more likely to occur at the positive maximum value of $\sigma_{L}$. This maximum value depends critically on the $\frac{r}{H}$ ratio, for a certain angle of rupture $\theta$, so it is possible, just by looking at the maxima of the solid curves in Fig. 6] (marked by solid points), to roughly match the height of the rupture point to the angle at which the breaking started to occur. As far as the actual prediction of the point of rupture, this would obviously depend on the building materials and the construction of the chimney or tower, an analysis of which goes beyond the scope of this work.

It is interesting to note, from Fig. [6 again, that the stress $\sigma_{L}$ is not always maximum at one third of the height (as for the bending moment of Fig. 5). For small angles of about $\theta \simeq 5^{\circ}-20^{\circ}$ it reaches a maximum for $\frac{r}{H} \simeq 0.4-0.5$, while for larger angles it approaches the typical ratio $\frac{r}{H} \simeq \frac{1}{3}$. This means that if the chimney breaks early in its fall, for small angles, it is more likely to break near the center; on the contrary if the rupture occurs at larger angles, the breaking point is usually shifted toward one third of the height. This is the effect of the $P_{r}$ term in Eq. 15, which modifies the position of the maximum $\sigma_{L}$ (or $\left.\sigma_{T}\right)$.

As already mentioned, another factor to be considered is the ratio $\frac{H}{a}$, which is usually in the range $\frac{H}{a} \simeq 5-20$ for real chimneys, but can be increased up to about $\frac{H}{a} \simeq 100$ with our toy models. In Fig. 7 we plot the maxima of the leading edge stress curves, for several values of the ratio $\frac{H}{a}=5,10,20,30, \ldots, 100$ (for the group of curves between the values of 30 and 100, the parameter is increased by 10 units for each curve).

These maxima are plotted as continuous curves, showing the corresponding values of the ratio $\frac{r}{H}$ and the angle $\theta$, at which the structure is more likely to break, for a given value of $\frac{H}{a}$. In other words, connecting for example the solid points of Fig. 6. we would obtain a corresponding continuous curve in Fig. 17, with the angle of probable rupture on the vertical axis, instead of the normalized stress.

The dependence of the rupture point on these quantities can be noted in several of the existing photos of falling chimneys, when the breaking occurs due to the bending of the structure and not for the transverse shear stress near the base mentioned at the beginning of this section. The angle of rupture can be roughly estimated by measuring the angle the upper part of the chimney forms with the vertical in the photos. This angle tends in fact not to change much after the rupture, since the upper part falls without much additional rotation. For example the photos in the paper by Bartlett ${ }^{5.21}$ refer to chimneys with $\frac{H}{a} \simeq 10$, breaking at about $\theta \simeq 20^{\circ}-25^{\circ}$ and for $\frac{r}{H} \simeq 0.47$, consistent with the values of Fig. 7. for the $\frac{H}{a}=10$ curve. Similar behavior can be seen in other photos,, 5.20 since real chimneys tend to break very early in their fall, due to the intense stresses originating within their structure.

\section{TOY MODELS}

In this section we discuss our efforts to reproduce the effects described above, with the help of toy models of the falling chimney. These models were constructed with simple toy blocks of different type and size, and their fall was filmed with a digital camera, so that we could analyze the events frame by frame, to test the theory. Complete details on the type of blocks used, experimental settings and video-capture techniques, as well as the complete set of our video-recordings and still photos can be found on our web-site,,$\frac{5}{-}$ and in an upcoming publication.$^{33}$ 


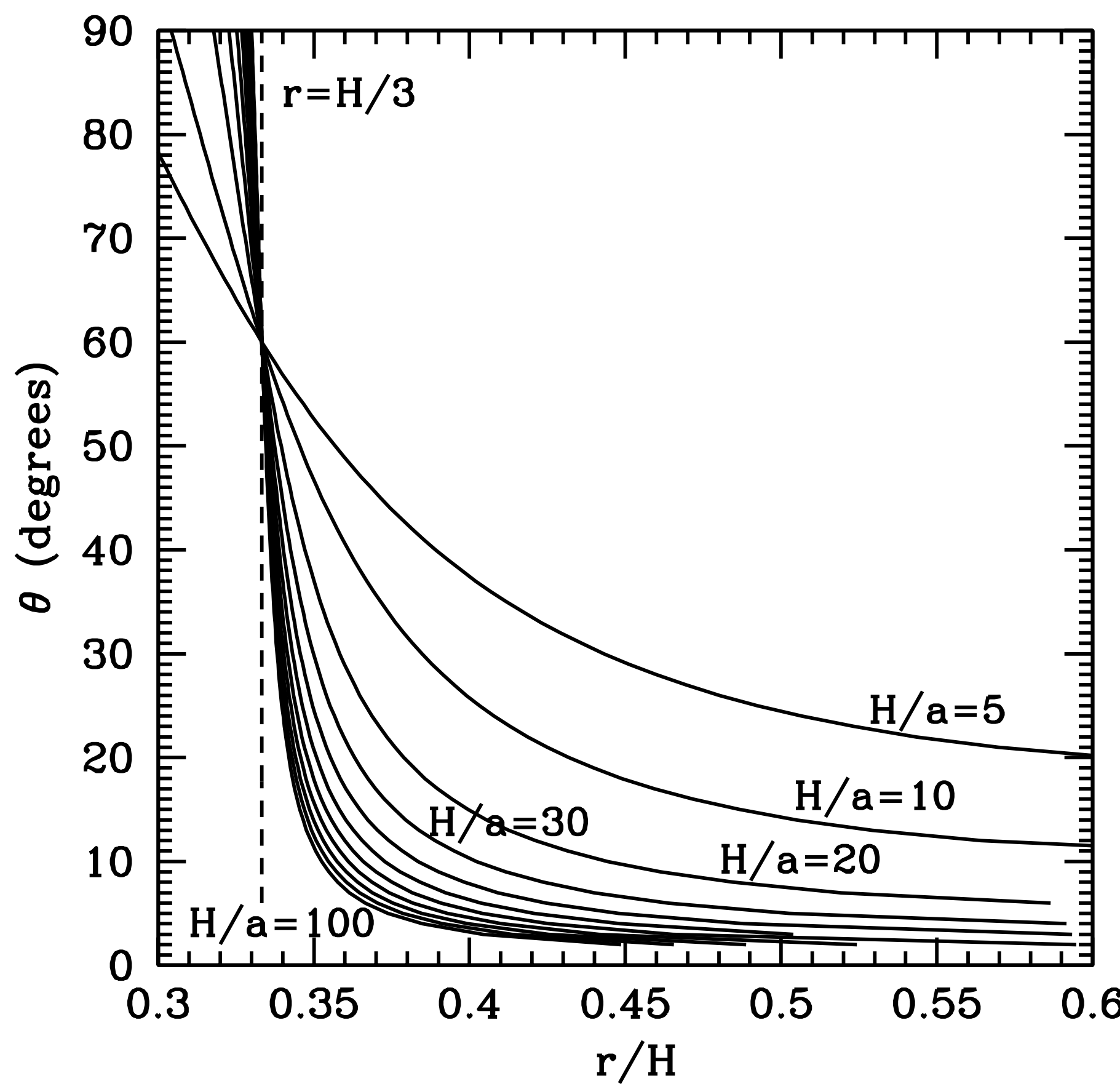

FIG. 7: The maxima of the leading edge stress curves are shown as continuous functions, for several values of the ratio $H / a$. They represent the points, in terms of the height ratio $r / H$ and angle $\theta$, at which the structure is more likely to break, due to bending. 


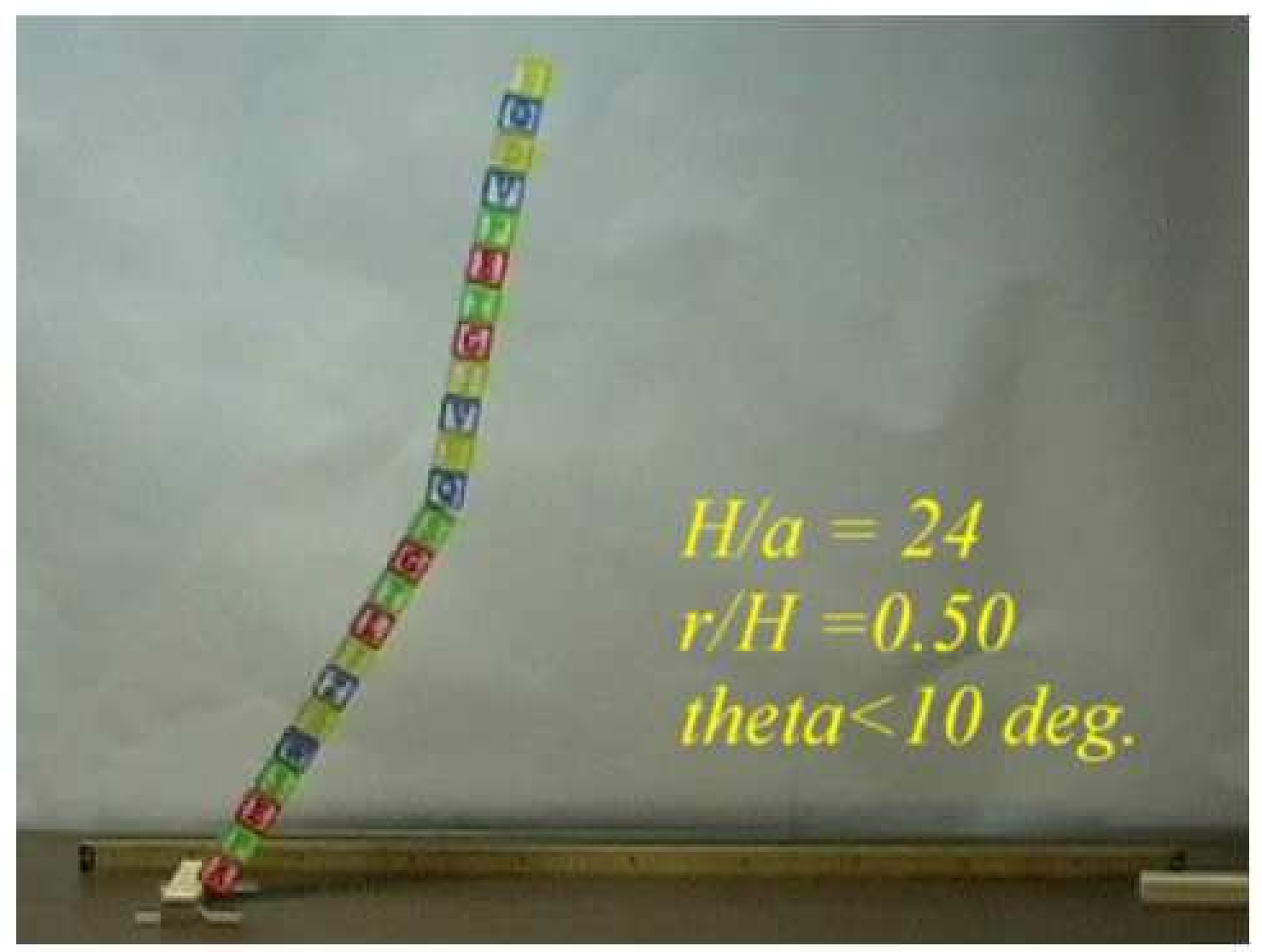

FIG. 8: The first toy model made with wooden blocks. The structure appears to break at $\frac{r}{H}=0.5$, and at a small angle $\theta \lesssim 10^{\circ}$, which can be estimated by measuring the angle formed by the upper part of the tower with the vertical.

Bundy noted in his work $^{20}$ that the use of a model to test a real chimney would be useless due to a "scale effect." The stresses inside the chimney depend roughly on the scale of the object, so that real chimneys would develop bigger stresses than equivalent smallscale models, therefore breaking earlier in their fall. Nevertheless we found it interesting to reproduce these effects in small scale models to test especially the discussion based on Figs. 6] and 7. It was also supposed to be difficult ${ }^{20}$ to show these effects with toy models. ${ }^{38}$

Fig. 8 is the first example of one of our toy models. We made a tower by simply stacking 24 wooden toy blocks of cubic shape, for a total height $H=0.76 \mathrm{~m}$, mass $m=0.32 \mathrm{~kg}$ and a ratio $\frac{H}{a}=24$, the value used in Fig. 6. The tower was set into the falling motion by removing a support at the bottom, inducing a rotation without slipping at the bottom point. The picture clearly shows the "rupture" due to bending of the structure at exactly half the height, $\frac{r}{H}=0.50$, and for a small angle $\theta \lesssim 10^{\circ}$, which again can be estimated by 


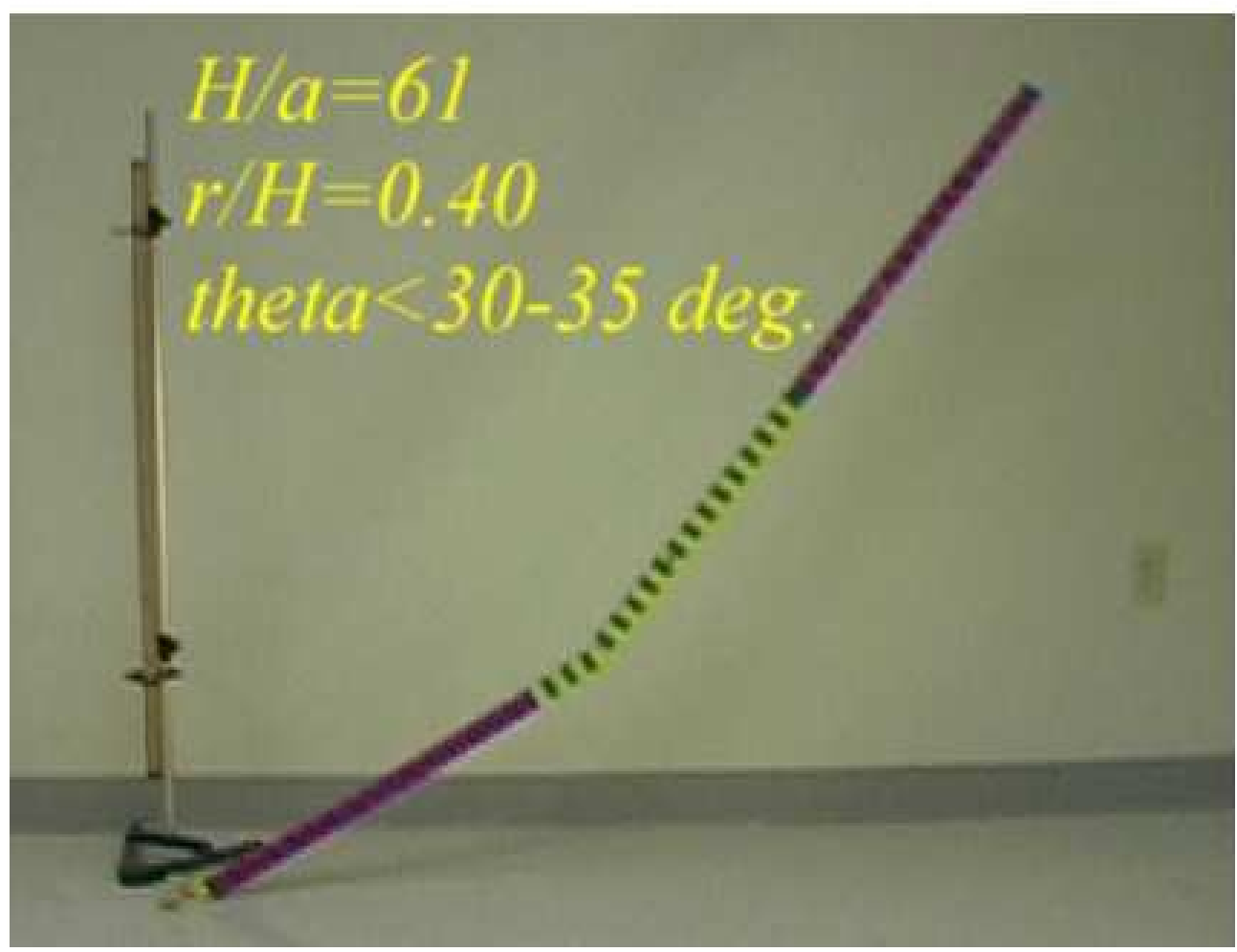

FIG. 9: The second toy model made with plastic blocks. The structure appears to break at $\frac{r}{H}=0.40$, and at a larger angle $\theta \simeq 30^{\circ}-35^{\circ}$, which can be estimated by measuring the angle formed by the upper part of the tower with the vertical.

measuring the angle the upper part of the chimney forms with the vertical direction. This is in good agreement with the position of the maximum for the solid- $10^{\circ}$ curve in Fig. [6] and also with the data of Fig. [7] using the $\frac{H}{a}=20$ curve, showing that the theory is applicable also to these small-scale models.

Our second example is a taller tower $\left(H=1.9 \mathrm{~m}, \mathrm{~m}=0.65 \mathrm{~kg}\right.$, and $\left.\frac{H}{a}=61\right)$ made with 100 plastic blocks of a very popular brand of toy bricks. The blocks are inserted on top of each other so that bending of the structure is allowed, but shear stress cannot possibly break the tower. The 100 toy blocks are arranged by color to subdivide the structure into three equal parts, and also the position of the center is marked. This time the rupture occurs for an angle around $\theta \simeq 30^{\circ}-35^{\circ}$, and at the height ratio $\frac{r}{H}=0.40$. This is consistent with the $30^{\circ}$ solid curve in Fig. 6, while the data from Fig. 7 (for $\frac{H}{a}=60$ ) would suggest a smaller angle of rupture. It is actually difficult, with this type of toy bricks, to estimate the angle 
at which the structure begins to bend.

We performed several other experiments, varying the dimensions of the towers, the type of blocks, always obtaining results consistent with the theory. We can conclude that it is actually easy to reproduce the bending and breaking of chimneys with small scale towers, and this type of experiment could be made part of an undergraduate laboratory class for rotational mechanics, with some minor adaptations and changes. ${ }^{33}$

\section{CONCLUSION}

In this paper we reviewed the theory of the falling chimney, showing that the rupture can be caused by either shear forces typically near the base, or by the bending of the structure which is caused primarily by the bending moment, but is also affected by the longitudinal stress force. In the latter case the breaking is more likely to occur between one third and one half of the height of the chimney.

This point of rupture is also related to the angle of rupture and this relationship can be verified in the many existing photographic reports of falling chimneys. We also constructed several small scale toy models, showing that it is possible with them to reproduce the dynamics of the fall. By examining photos taken during the fall of these models we were able to confirm the theoretical model outlined in this paper.

\section{Acknowledgments}

This research was supported by an award from Research Corporation. The authors would like to thank Dr. V. Coletta for the many useful discussions regarding this paper. G.V. would like to acknowledge and thank his friend and physics teacher, Prof. G. Tonzig, whose excellent book ${ }^{34}$ "Cento Errori di Fisica" inspired the original idea for this paper.

* Electronic address: gvarieschi@lmu.edu; URL: http://myweb.lmu.edu/gvarieschi/

$\dagger$ Electronic address: kkamiya@lion.lmu.edu

1 R. M. Sutton, Demonstration Experiments in Physics. (McGraw-Hill, New York, USA, 1938).

2 W. A. Hilton, Physics Demonstration Experiments at William Jewell College. (William Jewell College, Liberty, USA, 1971).

3 G. D. Freier and F. J. Anderson, A Demonstration Handbook for Physics. (American Association of Physics Teachers, College Park, USA, 1981).

4 D. R. Carpenter and R. B. Minnix, The Dick and Rae Physics Demo Notebook. (Dick and Rae Inc., Lexington, USA, 1993).

5 K. Kamiya and G. Varieschi, The Falling Chimney Web Page. (http://myweb.lmu.edu/gvarieschi/chimney/chimney.html).

6 P. A. Constantinides, "Experiments on torque, angular acceleration and moment of inertia," Am. J. Phys. 7 (1939) 254-257.

7 C. A. Ludeke, "Experimental examples in dynamics," Am. J. Phys. 9 (1941) 162-166.

8 W. A. Hilton, "Free fall paradox," Phys. Teach. 3 (1965) 323-324.

9 W. M. Young, "Faster than gravity!," Am. J. Phys. 52 (1984) 1142-1143. 
10 F. M. Phelps and L. R. Clifford, "How the ant got into the dish," Phys. Teach. 1 (1986) 293-294.

11 W. F. Theron, "The faster than gravity demonstration revisited," Am. J. Phys. 56 (1988) 736-739.

12 H. Hartel, "The falling stick with a greater than g," Phys. Teach. 38 (2000) 54-55.

13 L. A. Bloomfield, How Things Work. (Wiley, New York, USA, 1997).

14 R. M. Sutton, "Some teasers for conclusion jumpers," Am. J. Phys. 21 (1953) 658.

15 J. S. Miller, "On demonstrating a classical problem in analytical mechanics," Am. J. Phys. 20 (1952) 455-456.

16 G. W. Ficken, "Falling faster than g," Am. J. Phys. 41 (1973) 1013-1015.

17 A. A. Bartlett, "Falling chimney apparatus modification," Phys. Teach. 13 (1975) 435-437.

18 J. L. Adams, "Acceleration greater than g," Phys. Teach. 20 (1982) 100-101.

19 U. Haber-Schaim, "On qualitative problems," Phys. Teach. 30 (1992) 260.

20 F. P. Bundy, "Stresses in freely falling chimneys and columns," J. Appl. Phys. 11 (1940) $112-123$.

21 A. A. Bartlett, "More on the falling chimney," Phys. Teach. 14 (1976) 351-353.

22 R. M. Sutton, "Concerning falling chimneys," Science 84 (1936) 246-247.

23 J. B. Reynolds, "Falling chimneys," Science 87 (1938) 186-188.

24 E. L. Madsen, "Theory of the chimney breaking while falling," Am. J. Phys. 45 (1977) $182-184$.

25 A. T. Jones, "The falling chimney," Am. J. Phys. 14 (1946) 275.

26 J. Walker, The Flying Circus of Physics with Answers. (Wiley, New York, USA, 1975).

27 S. B. Cahn and B. E. Nadgorny, A Guide to Physics Problems - part 1. (Plenum Press, New York, USA, 1994).

28 J. A. Cronin and V. L. Telegdi, Graduate Problems in Physics. (Univ. of Chicago Press, Chicago, USA, 1979).

29 R. C. Hibbeler, Structural Analysis. (Prentice Hall, Upper Saddle River, N.J., USA, 2002).

30 R. C. Hibbeler, Mechanics of Materials. (Prentice Hall, Upper Saddle River, N.J., USA, 1997).

31 A. Sommerfeld, Mechanics of Deformable Bodies. (Academic Press, New York, USA, 1950).

32 L. D. Landau and E. M. Lifshitz, Theory of Elasticity. (Pergamon Press, New York, USA, 1986).

33 G. Varieschi. (in preparation).

34 G. Tonzig, Cento Errori di Fisica. (Sansoni, Bologna, Italy, 1991).

35 This is in general a good approximation. For a right circular hollow cylinder of outer and inner radii $r_{1}, r_{2}$ and length $H$, hinged at one end, the moment of inertia is $I=m\left(\frac{r_{1}^{2}+r_{2}^{2}}{4}+\frac{1}{3} H^{2}\right) \simeq \frac{1}{3} m H^{2}$ for $r_{1}, r_{2} \ll H$. For a typical chimney $r_{1} \lesssim \frac{H}{10}$, thus $\frac{r_{1}^{2} / 4}{H^{2} / 3} \lesssim 0.0075$, i.e., a correction of less than one percent. The correction due to $r_{2}$ is even smaller.

36 The bending moment and the stress forces can be thought as applied to the center (centroid) of the cross sectional area we are considering, on the face belonging to the lower portion of the structure. Equal and opposite forces and moments would originate on the face belonging to the upper portion.

37 A positive sign in the relation between bending moment and shear is usually reported in textbooks on Statics, due to a different choice of the sign of the moment.

38 We did not find any use of toy models for this problem, either in the literature or in web-pages 
devoted to physics demos. We will be grateful to receive information about similar experiments, if any. 